\title{
The Characteristic Solutions to the V-Notch Plane Problem of Anisotropy and the Associated Finite Element Method
}

\author{
Ge Tian, ${ }^{1}$ Xiang-Rong Fu, ${ }^{1}$ Ming-Wu Yuan, ${ }^{2}$ and Meng-Yan Song ${ }^{1}$ \\ ${ }^{1}$ Department of Civil Engineering, China Agricultural University, Beijing 100083, China \\ ${ }^{2}$ Department of Mechanics and Engineering Science, Peking University, Beijing 100871, China \\ Correspondence should be addressed to Xiang-Rong Fu; fuxr@cau.edu.cn
}

Received 17 July 2013; Accepted 13 August 2013

Academic Editor: Song Cen

Copyright (c) 2013 Ge Tian et al. This is an open access article distributed under the Creative Commons Attribution License, which permits unrestricted use, distribution, and reproduction in any medium, provided the original work is properly cited.

\begin{abstract}
This paper presents a novel way to calculate the characteristic solutions of the anisotropy V-notch plane problem. The material eigen equation of the anisotropy based on the Stroh theory and the boundary eigen equation of the V-notch plane problem are studied separately. A modified Müller method is utilized to calculate characteristic solutions of anisotropy V-notch plane problem, which are employed to formulate the analytical trial functions (ATF) in the associated finite element method. The numerical examples show that the proposed subregion accelerated Müller method is an efficient method to calculate the solutions of the equation involving the complex variables. The proposed element ATF-VN based on the analytical trial functions, which contain the characteristic solutions of the anisotropy V-notch problem, presents good performance in the benchmarks.
\end{abstract}

\section{Introduction}

The characteristic solutions to the V-notch plane problem were studied by many researchers [1-12]. Fu et al. studied the finite element method based on the analytical trial functions of the V-notch plane problem of isotropy $[2,3]$. Niu et al. studied the boundary element method based on the analytical solutions of the V-notch plane problem [4-6]. Ping et al. discussed the finite element of the V-notch plate problem [7].

This paper presents a novel way to calculate the characteristic solutions of the anisotropy V-notch plane problem and to study the finite element method associated with these solutions.

Firstly, the material eigen equation of the anisotropy based on the Stroh theory $[1,8-11]$ is studied, and the eigenvalues of the anisotropy are calculated.

Secondly, the calculated eigenvalues of material are employed to calculate the boundary eigenvalues of the Vnotch plane problem.

Thirdly, the paper proposed a novel modified Müller method, named as the subregion accelerated müller (SRAM) method, which is utilized to calculate characteristic solutions of anisotropy V-notch plane problem.
At last, the calculated eigenvalues of the V-notch plane problem are employed to formulate the analytical trial functions (ATF) in the associated finite element method.

In the numerical examples, the proposed subregion accelerated Müller method [2,3] is shown as an efficient method to calculate the solutions of the equation involving the complex variables. The proposed ATF-VN based on the analytical trial functions, which contain the characteristic solutions of the V-notch problem, presents good performance in the benchmarks.

Though many researchers studied the V-notch problem of isotropy material [1-16], there are seldom studies about the Vnotch problem of anisotropy material. This paper presents a novel systematic strategy to calculate the V-notch problem of anisotropy material.

\section{The Material Characteristic Matrix of Anisotropic}

The constitutive and equilibrium equations of anisotropy can be written as [1]

$$
\begin{gathered}
\sigma_{i j}=C_{i j k l} u_{k, l}, \\
\sigma_{i j, j}=C_{i j k l} u_{k, l j}=0,
\end{gathered}
$$


TABLE 1: The comparison of the convergence properties of several iterative methods.

\begin{tabular}{lcccccccccccccc}
\hline$a$ & $b$ & \multicolumn{2}{c}{ Direct (31) } & $n$ & \multicolumn{2}{c}{ SBM (34) } & $n$ & RDM (33) & & $n$ & SRAM (35) & $n$ \\
\hline 0.6 & 0.9 & 0.730900742 & 0 & 8 & 0.730900742 & 0 & 9 & 0.730900742 & 0 & 7 & 0.730900742 & 0 & 7 \\
1.8 & 2.1 & 1.89349155 & 0 & F & 2.0748262 & 0.2294258 & 20 & 2.00933205 & 0 & F & 2.0748262 & 0.2294258 & 30 \\
3 & 3.3 & 3.01938419 & 0 & F & 3.27976709 & 0.326690313 & 16 & 4.48290004 & -0.38898376 & 23 & 3.27976709 & -0.326690313 & 16 \\
4.2 & 4.5 & 3.27976709 & -0.32669031 & 19 & 4.48290004 & 0.388983761 & 16 & 4.48290004 & 0.388983761 & 9 & 4.48290004 & -0.388983761 & 17 \\
5.4 & 5.7 & 3.01938419 & $6.21124 E-13$ & $\mathrm{~F}$ & 3.27976709 & -0.32669031 & 51 & 9.28902685 & -0.5301614 & 25 & 5.68509375 & -0.435407938 & 20 \\
6.6 & 6.9 & 6.88672925 & 0.472546839 & 87 & 3.27976709 & -0.32669031 & 45 & 9.28902685 & 0.530161398 & 31 & 6.88672925 & -0.472546839 & 25 \\
\hline
\end{tabular}

TABLE 2: Characteristic eigenvalues of V-notch problem.

\begin{tabular}{|c|c|c|c|c|c|c|c|c|}
\hline \multicolumn{9}{|c|}{$2 \alpha$} \\
\hline & $190^{\circ}$ & $200^{\circ}$ & $210^{\circ}$ & $240^{\circ}$ & $270^{\circ}$ & $300^{\circ}$ & $330^{\circ}$ & $360^{\circ}$ \\
\hline$\xi_{1}$ & 0.93265944 & 0.87284430 & 0.81861307 & 0.67723697 & 0.55658245 & 0.50800061 & 0.50068400 & 0.50000000 \\
\hline$\xi_{2}$ & 1.86475055 & 1.74191187 & 1.62613243 & 1.28206691 & 0.88272695 & 0.65597019 & 0.56189499 & 0.50000000 \\
\hline
\end{tabular}

where $u_{i}$ is displacement, $\sigma_{i j}$ is stress, and $C_{i j k l}$ is the elastic tensor of the anisotropy, in which $i, j, k, l=1,2,3$ are denoted as coordinates $x_{i}(i=1,2,3)$ of the three-dimensional problem.

In the plane problem of anisotropy, the displacements $\mathbf{u}_{k}(k=1,2,3)$ are assumed to be only associated with the coordinates $x_{1}$ and $x_{2}$. According to Stroh theory $[8,9]$,

$$
\mathbf{u}_{k}=\mathbf{a}_{k} g\left(z_{k}\right),
$$

in which $g$ is the analytic function of $z_{k}$, while $z_{k}=x_{1}+p_{k} x_{2}$. $\mathbf{a}_{k}$ is the eigenvector about the eigenvalue $p_{k}$ of the material.

Substituting (3) into (2), we have

$$
\left[\mathbf{Q}+\left(\mathbf{R}+\mathbf{R}^{T}\right) p_{k}+\mathbf{T} p_{k}^{2}\right] \mathbf{a}_{k} g^{\prime \prime}\left(z_{k}\right)=\mathbf{D} \mathbf{a}_{k} g^{\prime \prime}\left(z_{k}\right)=0
$$

in which

$$
\mathbf{D}=\left[\mathbf{Q}+\left(\mathbf{R}+\mathbf{R}^{T}\right) p_{k}+\mathbf{T} p_{k}^{2}\right]
$$

The material coefficient matrixes of $\mathbf{Q}, \mathbf{R}$, and $\mathbf{T}$ are defined as $Q_{i k}=C_{i 1 k 1}$, and $R_{i k}=C_{i 1 k 2}, T_{i k}=C_{i 2 k 2}$. They can also be denoted as

$$
\begin{aligned}
& \mathbf{Q}=\left[\begin{array}{lll}
C_{11} & C_{16} & C_{15} \\
C_{61} & C_{66} & C_{65} \\
C_{51} & C_{56} & C_{55}
\end{array}\right], \\
& \mathbf{R}=\left[\begin{array}{lll}
C_{16} & C_{12} & C_{14} \\
C_{66} & C_{62} & C_{64} \\
C_{56} & C_{52} & C_{54}
\end{array}\right], \\
& \mathbf{T}=\left[\begin{array}{lll}
C_{66} & C_{62} & C_{64} \\
C_{26} & C_{22} & C_{24} \\
C_{46} & C_{42} & C_{44}
\end{array}\right] .
\end{aligned}
$$

In order to obtain the nonzero solutions of (4), the determinant of the coefficient matrix $\mathbf{D}$ must be zero:

$$
\operatorname{det} \mathbf{D}=0
$$

The solutions $p_{k}(k=1,2,3)$ of $(7)$ are the eigenvalues of the material.

In the same way, the stresses, which satisfied equilibrium equations, can also be expressed by the characteristic solutions of stress functions $\phi_{k}$, and we have

$$
\begin{array}{cc}
\phi_{k}=\mathbf{b}_{k} f\left(z_{k}\right) & (k=1,2,3), \\
\sigma_{i 1}=-\partial \phi_{i, 1}, & \sigma_{i 2}=\partial \phi_{i, 2},
\end{array}
$$

where $f$ is the first derivative of $g$.

According to (1), we have

$$
\mathbf{b}_{k}=\left(\mathbf{R}^{T}+p_{k} \mathbf{T}\right) \mathbf{a}_{k}=-\frac{1}{p_{k}}\left(\mathbf{Q}+p_{k} \mathbf{R}\right) \mathbf{a}_{k}
$$

The general solutions of displacement in (1) can be denoted as

$$
\mathbf{u}=\mathbf{A} \cdot \mathbf{g}
$$

in which $\mathbf{A}=\left[\mathbf{a}_{1}, \mathbf{a}_{2}, \mathbf{a}_{3}\right]$.

The general solutions $\boldsymbol{\Phi}=\left[\phi_{1}, \phi_{2}, \phi_{3}\right]^{T}$ in (8) of stress functions can be denoted as

$$
\Phi=\mathbf{B} \cdot \mathbf{f}
$$

in which $\mathbf{B}=\left[\mathbf{b}_{1}, \mathbf{b}_{2}, \mathbf{b}_{3}\right]$. 


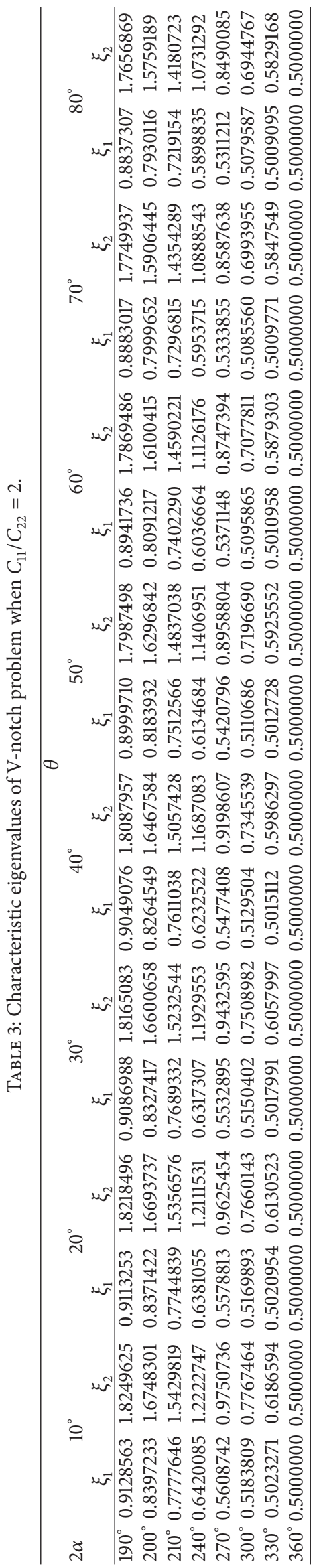




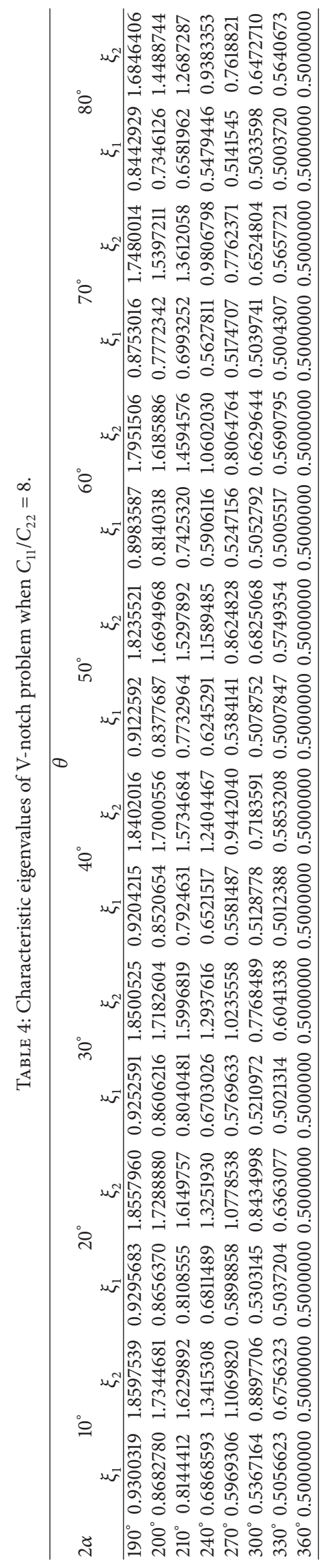




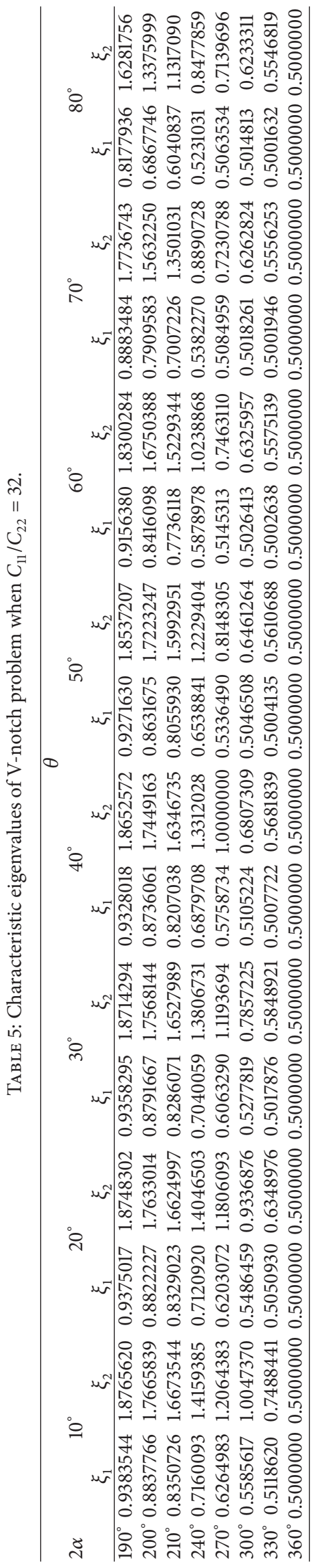


Introducing the normalization condition

$$
\begin{aligned}
& 2 \mathbf{a}_{k}^{T} \mathbf{b}_{k}=1,
\end{aligned}
$$

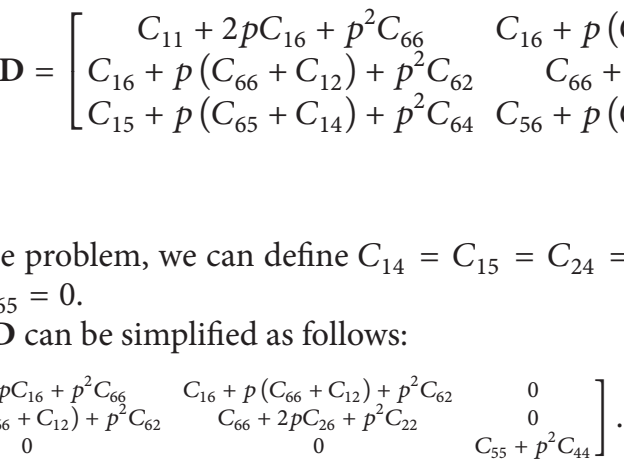

So matrix $\mathbf{D}$ can be simplified as follows: the characteristic matrix of the material $\mathbf{A}=\left[\mathbf{a}_{1}, \mathbf{a}_{2}, \mathbf{a}_{3}\right]$ and $\mathbf{B}=\left[\mathbf{b}_{1}, \mathbf{b}_{2}, \mathbf{b}_{3}\right]$ can be determined.

Substituting (6) into (5), we can get $C_{25}=C_{64}=C_{65}=0$.

$\mathbf{D}=\left[\begin{array}{ccc}C_{11}+2 p C_{16}+p^{2} C_{66} & C_{16}+p\left(C_{66}+C_{12}\right)+p^{2} C_{62} & 0 \\ C_{16}+p\left(C_{66}+C_{12}\right)+p^{2} C_{62} & C_{66}+2 p C_{26}+p^{2} C_{22} & 0 \\ 0 & 0 & C_{55}+p^{2} C_{44}\end{array}\right]$.

The characteristic matrix A of the material can be written as

$\mathbf{A}=\left[\begin{array}{ccc}k_{1}\left(C_{66}+2 p_{1} C_{26}+p_{1}^{2} C_{22}\right) & k_{2}\left(C_{66}+2 p_{2} C_{26}+p_{2}^{2} C_{22}\right) & 0 \\ -k_{1}\left(C_{16}+p_{1}\left(C_{66}+C_{12}\right)+p_{1}^{2} C_{26}\right) & -k_{2}\left(C_{16}+p_{2}\left(C_{66}+C_{12}\right)+p_{2}^{2} C_{26}\right) & 0 \\ 0 & 0 & k_{3}\end{array}\right]$

The characteristic matrix $\mathbf{B}=\left[\mathbf{b}_{1}, \mathbf{b}_{2}, \mathbf{b}_{3}\right]$ can be calculated as

$$
\begin{gathered}
\mathbf{b}_{1}=\left[\begin{array}{c}
k_{1} p_{1}\left(C_{16} C_{26}+p_{1} C_{16} C_{22}+p_{1}^{2} C_{66} C_{22}-C_{66} C_{12}-p_{1} C_{26} C_{12}-p_{1}^{2} C_{26}^{2}\right) \\
k_{1}\left(C_{66} C_{12}+p_{1} C_{26} C_{12}+p_{1}^{2} C_{26}^{2}-C_{16} C_{26}-p_{1} C_{16} C_{22}-p_{1}^{2} C_{66} C_{22}\right) \\
0
\end{array}\right], \\
\mathbf{b}_{2}=\left[\begin{array}{c}
k_{2} p_{2}\left(C_{16} C_{26}+p_{2} C_{16} C_{22}+p_{2}^{2} C_{66} C_{22}-C_{66} C_{12}-p_{2} C_{26} C_{12}-p_{2}^{2} C_{26}^{2}\right) \\
k_{2}\left(C_{66} C_{12}+p_{2} C_{26} C_{12}+p_{2}^{2} C_{26}^{2}-C_{16} C_{26}-p_{2} C_{16} C_{22}-p_{2}^{2} C_{66} C_{22}\right) \\
0
\end{array}\right],
\end{gathered}
$$$$
\mathbf{b}_{3}=\left[\begin{array}{c}
0 \\
0 \\
k_{3} p_{3} C_{44}
\end{array}\right]
$$

The constants $k_{1}, k_{2}$, and $k_{3}$ in (16) and (17) can be determined by (13).

It can be proved that according to the characteristic matrixes A and B of anisotropy defined in (16) and (17), we have

$$
\begin{aligned}
& \mathbf{A A}^{T}+\overline{\mathbf{A}} \overline{\mathbf{A}}^{T}=\mathbf{0}=\mathbf{B} \mathbf{B}^{T}+\overline{\mathbf{B}} \overline{\mathbf{B}}^{T} \\
& \mathbf{A}^{T} \mathbf{B}+\mathbf{B}^{T} \mathbf{A}=\mathbf{I}=\overline{\mathbf{A}}^{T} \overline{\mathbf{B}}+\overline{\mathbf{B}}^{T} \overline{\mathbf{A}} .
\end{aligned}
$$

\section{The Boundary Characteristic Matrix of V-Notch}

The plane polar coordinate system $(r, \theta)$ was defined in Figure 1, and the $r$-axis divided the angle of the $\mathrm{V}$-notch into two equal parts.

As showed in Figure 1, in the plane V-notch problem of anisotropic, the notch tip is defined as the origin, and there is an angle $\theta$ between the $r$-axis and the material principle axis of $x_{1}$. So the material matrix of anisotropic material can be redefined as

$$
\mathbf{C}^{\prime}=\mathbf{T C T}^{T}
$$

in which $\mathbf{T}$ is the transformation matrix, and we have

$$
\mathbf{T}=\left[\begin{array}{ccc}
\cos ^{2} \theta & \sin ^{2} \theta & -2 \sin \theta \cos \theta \\
\sin ^{2} \theta & \cos ^{2} \theta & 2 \sin \theta \cos \theta \\
\sin \theta \cos \theta & -\sin \theta \cos \theta & \cos ^{2} \theta-\sin ^{2} \theta
\end{array}\right]
$$

In the polar coordinate system, $z_{k}$ can be expressed as

$$
z_{k}=r \zeta_{k}(\theta)
$$

in which

$$
\zeta_{k}(\theta)=\cos (\theta)+p_{k} \sin (\theta)
$$

$r$ represents the radial distance to tip.

The general stress solutions $f\left(z_{k}\right)$ in (8) can be expressed by $f\left(r, \zeta_{k}(\theta)\right)$. According to Stroh's theory, vector $\mathbf{f}$ can be written as $[10,11]$

$$
\mathbf{f}=\frac{c}{2}\left(\frac{r}{\hat{r}}\right)^{\lambda} \Lambda(\theta, \alpha, \lambda) \mathbf{q}+\frac{\bar{c}}{2}\left(\frac{r}{\hat{r}}\right)^{\bar{\lambda}} \boldsymbol{\Lambda}(\theta, \alpha, \bar{\lambda}) \overline{\mathbf{q}}
$$

in which eigenvalues $\lambda$ and parameter $c$ are a complex constant, eigenvector $\mathbf{q}$ is undetermined complex vector, are $\widehat{r}$ is reference length of the notch, and we have

$$
\boldsymbol{\Lambda}(\theta, \alpha, \lambda)=\operatorname{diag}\left[\left(\frac{\zeta_{1}(\theta)}{\zeta_{1}(\alpha)}\right)^{\lambda},\left(\frac{\zeta_{2}(\theta)}{\zeta_{2}(\alpha)}\right)^{\lambda},\left(\frac{\zeta_{3}(\theta)}{\zeta_{3}(\alpha)}\right)^{\lambda}\right] \mathbf{B}^{T}
$$

Taking account the stress $\mathbf{t}_{r}$ in the boundaries of the Vnotch, where $\theta= \pm \alpha$,

$$
\mathbf{t}_{r}=-\frac{\partial \Phi}{\partial r}
$$
notch

Generally $\mathbf{t}_{r}$ is zero in the stress-free boundaries of the V-

Utilizing (18), $\mathbf{t}_{r}$ is zero in the boundary $\theta=\alpha$.

Considering the condition of $(r / \widehat{r})^{\lambda-1} \neq 0$, in order to make $\mathbf{t}_{r}$ equal to zero on the side $\theta=-\alpha$, according to (24), we have

$$
c \lambda(\mathbf{B} \mathbf{\Lambda}(-\alpha, \alpha, \lambda)+\overline{\mathbf{B} \boldsymbol{\Lambda}(-\alpha, \alpha, \bar{\lambda})}) \mathbf{q}=c \lambda \mathbf{C q}=\mathbf{0},
$$

in which the boundary characteristic matrix $\mathbf{C}$ is

$$
\mathbf{C}=\mathbf{B} \boldsymbol{\Lambda}(-\alpha, \alpha, \lambda)+\overline{\mathbf{B} \boldsymbol{\Lambda}(-\alpha, \alpha, \bar{\lambda})}
$$


In order to obtain the nonzero solutions of (27), the determinant of the coefficient matrix $\mathbf{C}$ must be zero:

$$
\Delta=|\mathbf{C}|=0 .
$$

Equation (29) is the boundary characteristic equation. Its solution $\lambda$ is complex; $\mathbf{u}$ and $\phi$ in (11) and (12) are also complex. If $\lambda$ is the root of the equation, $\bar{\lambda}$ is also the root of the equation.

\section{The Müller Method Accelerated by Subregion}

The Müller method is an effective method in solving the zero point calculation. As showed in Figure 2, making a parabola $g(z)$ which passes three points $z_{i}, z_{i-1}, z_{i-2}$ on the complex function $f(z)$, we have

$$
g(z)=f\left(z_{i}\right)+\left(z-z_{i}\right) a_{i}+f\left[z_{i}, z_{i-1}, z_{i-2}\right]\left(z-z_{i}\right)^{2},
$$

where $f\left[z_{i}, z_{i-1}\right], f\left[z_{i}, z_{i-1}, z_{i-2}\right]$ is the first and second difference of $f(z)$.

The root of the $g(z)=0$ is

$$
z^{*}=z_{i}+\frac{-a_{i} \pm \sqrt{a_{i}^{2}-4 f\left(z_{i}\right) f\left[z_{i}, z_{i-1}, z_{i-2}\right]}}{2 f\left[z_{i}, z_{i-1}, z_{i-2}\right]},
$$

in which

$$
a_{i}=f\left[z_{i}, z_{i-1}\right]+f\left[z_{i}, z_{i-1}, z_{i-2}\right]\left(z_{i}-z_{i-1}\right) .
$$

In the next iteration step, $z_{i+1}=z^{*}$ is the new initial value.

To solve more than one root, there is an effective modification (RDM) to the function $f(z)$

$$
f_{n}(z)=\frac{f(z)}{\left(z-z_{1}^{0}\right)\left(z-z_{2}^{0}\right) \cdots\left(z-z_{n}^{0}\right)},
$$

where $z_{1}^{0}, z_{2}^{0}, \ldots, z_{n}^{0}$ are series of characteristic roots which have been calculated.

The Müller method is local convergence iteration method, if the root of equation $f(z)=0$ is confirmed in the interval of $a$ and $b$. There is an effective method to accelerate the convergence of the Müller method

$$
f^{*}(z)=\frac{f(z)}{(z-a)(b-z)} .
$$

It is called Shrink Boundary Method (SBM).

Combining (33) with (34), we have the subregion accelerated müller (SRAM) method, whose iteration function is

$$
f_{n}^{*}(z)=\frac{f(z)}{(z-a)(b-z)\left(z-z_{1}^{0}\right) \cdots\left(z-z_{n}^{0}\right)} .
$$

Figure 3 shows the values of $f(z)$ and $f^{*}(z)$ near the root, where the subregion of the iteration is $a=0.6$ and $b=0.9$, $f(z)=z \cdot \sin (A)-\sin (z \cdot A), A=300^{\circ}$.

In Figure 3, to the values of the tangent near root, $f^{*}(z)$ is larger than $f(z)$ due to the SBM.

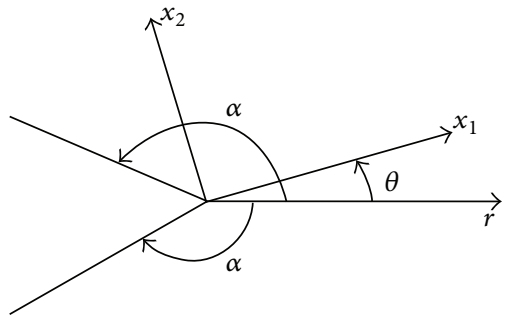

FIgURE 1: The local coordinators.

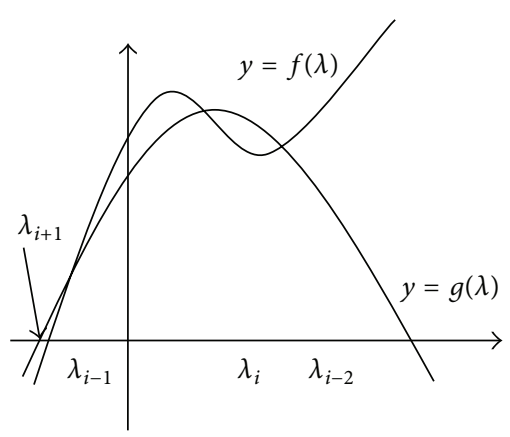

FIgURE 2: The schematic of Müller method.

Table 1 shows the results of the eigenvalues to $f(z)$ in four different Müller methods (Direct, SBM, RDM, and SRAM). The value $n$ in Table 1 presents the times of iteration ( $F$ means fail to converge). SRAM shows very good performance in the calculation of the eigenvalues. The SBM and the SRAM can reach the convergence results in every subregion, but the direct Müller method and the RDM fail to converge in some subregions. SRAM can get rid of the influence of the eigenvalues calculated before the step and converge faster than other methods in most cases.

\section{The Element ATF-VN Based on the Analytical Trial Functions}

According to the subregion mixed energy principle, the total energy can be written as

$$
\Pi=\Pi_{P}-\Pi_{C}+H_{P C},
$$

where $\Pi_{C}$ is the complementary energy in the subregion- $C$ that was defined by the stress field, $\Pi_{P}$ is the potential energy in the subregion- $P$ that was defined by the displacement field, and $H_{P C}$ is the additional energy along the boundary between two subregions.

The potential energy $\Pi_{P}$ can be denoted as

$$
\Pi_{P}=\frac{1}{2}\{\mathbf{w}\}^{T}\left[\mathbf{K}_{\mathbf{O}}\right]\{\mathbf{w}\}-\{\mathbf{w}\}^{T}\{\mathbf{P}\}
$$

in which $\{\mathbf{w}\}$ is the nodal displacement vector, $\{\mathbf{P}\}$ is the equivalent nodal load vector, and $\left[\mathbf{K}_{\mathbf{O}}\right]$ is the stiffness matrix of the potential energy in subregion- $P$ (SRP).

The complementary energy $\Pi_{c}$ can be denoted as

$$
\Pi_{c}=\frac{1}{2}\{\boldsymbol{\beta}\}^{T}[\mathbf{V}]\{\boldsymbol{\beta}\}
$$




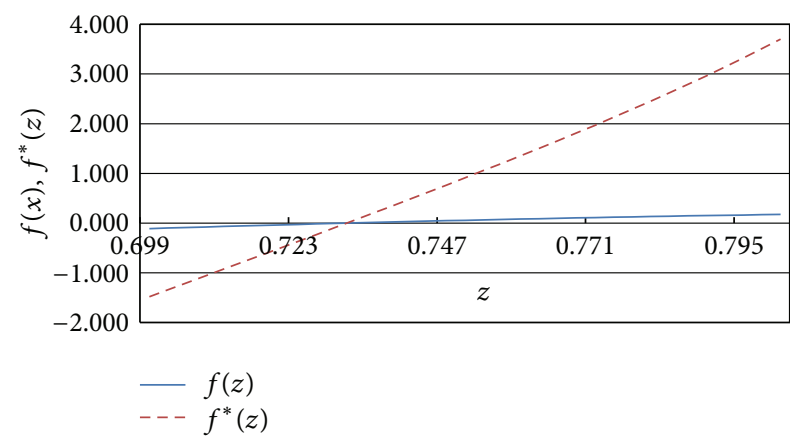

Figure 3: Values of $f(z)$ and $f^{*}(z)$ near the root.
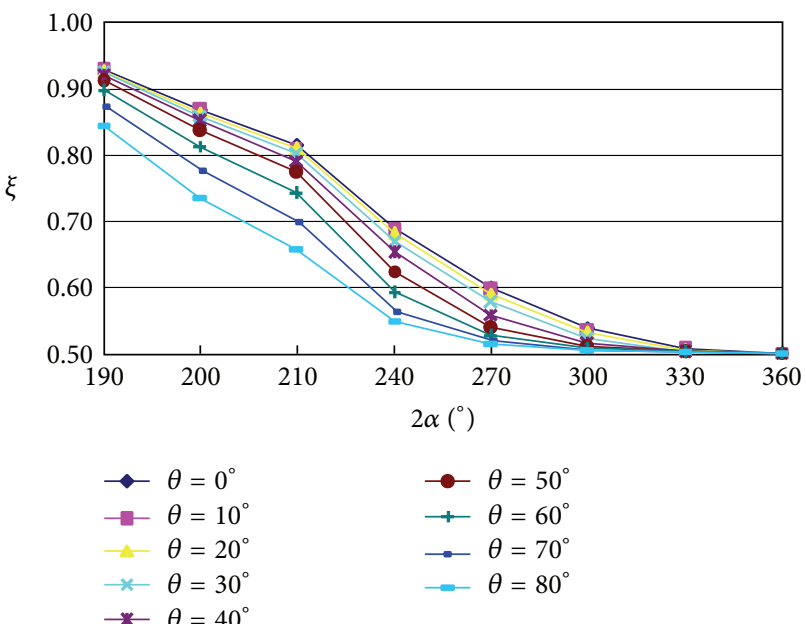

FIgURE 4: The first eigenvalue while $C_{11} / C_{22}=8$.

in which $\{\boldsymbol{\beta}\}$ is the undetermined parameters of the stress, and

$$
[\mathbf{V}]=\iint_{\Omega_{\mathrm{C}}}[\mathbf{S}]^{T}[\mathbf{D}]^{-1}[\mathbf{S}] t d A,
$$

where [D] is elastic coefficient matrix, $t$ is thickness, and $[\mathbf{S}]$ is the stress trial functions of the complementary energy subregion- $C$ (SRC), which is defined in (9).

The additional energy $H_{P C}$ on the boundary $\Gamma$ between two kinds of subregions can be written as

$$
H_{P C}=\int_{\Gamma}\{\mathbf{T}\}^{T}\{\overline{\mathbf{u}}\} t d s
$$

in which $\{\mathbf{T}\}$ is the boundary force determined in SRC:

$$
\{\mathbf{T}\}=[\mathbf{L}]\{\boldsymbol{\sigma}\}=[\mathbf{L}][\mathbf{S}]\{\boldsymbol{\beta}\},
$$

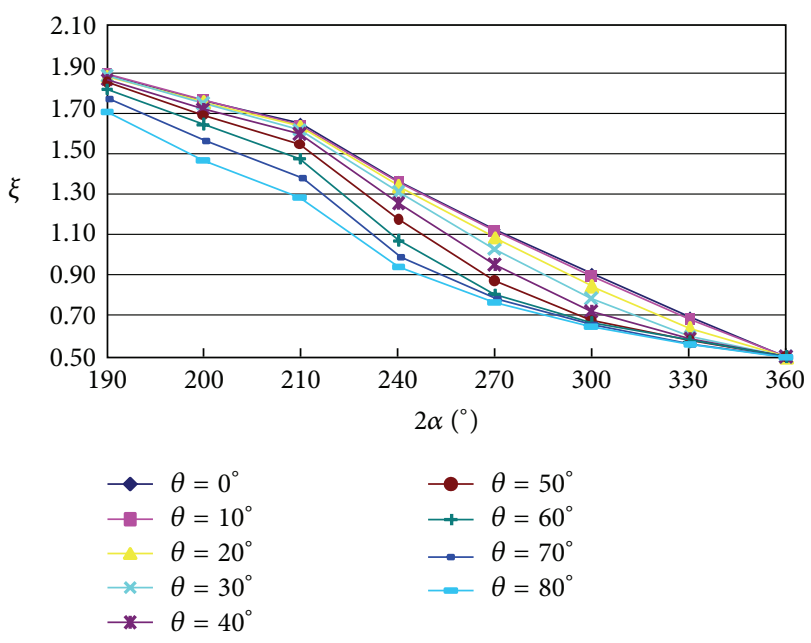

\begin{tabular}{|c|c|c|}
\hline & \multicolumn{2}{|c|}{$2 \alpha$} \\
\hline & $330^{\circ}$ & $350^{\circ}$ \\
\hline$\xi_{1}$ & 0.50145301 & 0.50005299 \\
\hline$\eta_{1}$ & 0 & 0 \\
\hline$\xi_{2}$ & 0.59819181 & 0.52935471 \\
\hline$\eta_{2}$ & 0 & 0 \\
\hline$\xi_{3}$ & 1 & 1 \\
\hline$\eta_{3}$ & 0 & 0 \\
\hline$\xi_{4}$ & 1.20295709 & 1.05884289 \\
\hline$\eta_{4}$ & 0 & 0 \\
\hline$\xi_{5}$ & 1.49037781 & 1.49972777 \\
\hline$\eta_{5}$ & 0 & 0 \\
\hline$\xi_{6}$ & 1.83893404 & 1.5886091 \\
\hline$\eta_{6}$ & 0 & 0 \\
\hline$\xi_{7}$ & 1.948556 & 1.99910697 \\
\hline$\eta_{7}$ & 0 & 0 \\
\hline$\xi_{8}$ & 2.44049194 & 2.11882272 \\
\hline$\eta_{8}$ & 0.11420683 & 0 \\
\hline$\xi_{9}$ & 2.98700495 & 2.49797992 \\
\hline$\eta_{9}$ & 0.16674099 & 0 \\
\hline$\xi_{10}$ & 3.53334548 & 2.64969856 \\
\hline$\eta_{10}$ & 0.20371063 & 0 \\
\hline$\xi_{11}$ & 4.07955465 & 2.99614098 \\
\hline$\eta_{11}$ & 0.2330165 & 0 \\
\hline$\xi_{12}$ & 4.62566114 & 3.18153251 \\
\hline$\eta_{12}$ & 0.25754031 & 0 \\
\hline$\xi_{13}$ & 5.17168563 & 3.71477243 \\
\hline$\eta_{13}$ & 0.27872957 & 0 \\
\hline$\xi_{14}$ & 6.26354629 & 3.98901973 \\
\hline$\eta_{14}$ & 0.31420638 & 0 \\
\hline
\end{tabular}

FIgURE 5: The second eigenvalue while $C_{11} / C_{22}=8$.

TABLE 6: The eigenvalues of the V-notch specimen. 
TABLE 7: The values of $K_{\text {II }}$ calculated by ATF-VN.

\begin{tabular}{lccccccc}
\hline$N$ items of the ATF & 4 & 6 & 8 & 10 & 12 & 14 & Reference [12] \\
\hline $350^{\circ}$ & 0.41956745 & 0.40362374 & 0.4003461 & 0.39869345 & 0.39876045 & 0.39877248 & 0.401 \\
$330^{\circ}$ & 0.15386682 & 0.12706163 & 0.12363552 & 0.12187221 & 0.12195786 & 0.12195786 & $/$ \\
\hline
\end{tabular}

where direction cosine matrix $[\mathbf{L}]$ can be written as

$$
[\mathbf{L}]=\left[\begin{array}{ccc}
\cos \theta \cos \gamma & \sin \theta \sin \gamma & -(\sin \theta \cos \gamma+\cos \theta \sin \gamma) \\
\sin \theta \cos \gamma & -\cos \theta \sin \gamma & \cos \theta \cos \gamma-\sin \theta \sin \gamma
\end{array}\right]
$$

$\gamma$ is the angle between the normal direction of $\Gamma$ and $r$-axis.

In (41), $\{\overline{\mathbf{u}}\}$ is the displacement on the boundary, which is defined in SRP:

$$
\{\overline{\mathbf{u}}\}=[\overline{\mathbf{N}}]\{\overline{\mathbf{w}}\},
$$

where $\{\overline{\mathbf{w}}\}$ is the nodal displacements on the boundary between two subregions and $\{\overline{\mathbf{N}}\}$ is the shape function defined in SRP.

Equation (40) can also be expressed as

$$
H_{P C}=\{\boldsymbol{\beta}\}^{T}[\mathbf{H}]\{\overline{\mathbf{w}}\},
$$

in which

$$
[\mathbf{H}]=\int_{T}[\mathbf{S}]^{T}[\mathbf{L}]^{T}[\overline{\mathbf{N}}] t d s .
$$

The total energy $\Pi$ can be denoted as

$$
\begin{aligned}
\Pi= & \frac{1}{2}\{\mathbf{w}\}^{T}\left[\mathbf{K}_{\mathbf{O}}\right]\{\mathbf{w}\}-\{\mathbf{w}\}^{T}\{\mathbf{P}\} \\
& -\frac{1}{2}\{\boldsymbol{\beta}\}^{T}[\mathbf{V}]\{\boldsymbol{\beta}\}+\{\boldsymbol{\beta}\}^{T}[\mathbf{H}]\{\overline{\mathbf{w}}\} .
\end{aligned}
$$

Using the stationary conditions of the total energy $\Pi$

$$
\delta \Pi=0
$$

the stiffness matrix of $\{\overline{\mathbf{w}}\}$ can be obtained as

$$
[\overline{\mathbf{K}}]=[\mathbf{H}]^{T}[\mathbf{V}]^{-1}[\mathbf{H}] .
$$

The generalized element defined in (48) is named as ATF$\mathrm{VN}$.

\section{Numerical Examples}

6.1. Example 1. This example shows the calculation of the characteristic solutions of a V-notch slab, which is made of anisotropic material T700, and its layer angle $\theta$ is $45^{\circ}$.

The material parameters matrix of T700 is

$$
\mathbf{C}=\left[\begin{array}{ccc}
1.38 \times 10^{11} & 4.14 \times 10^{9} & 0 \\
4.14 \times 10^{9} & 9.79 \times 10^{9} & 0 \\
0 & 0 & 4.47 \times 10^{9}
\end{array}\right]
$$

The layer angle $\theta$ is $45^{\circ}$. According to (21) and (20)

$$
\begin{gathered}
\mathbf{T}=\left[\begin{array}{ccc}
\cos ^{2} 45^{\circ} & \sin ^{2} 45^{\circ} & -2 \sin 45^{\circ} \cos 45^{\circ} \\
\sin ^{2} 45^{\circ} & \cos ^{2} 45^{\circ} & 2 \sin 45^{\circ} \cos 45^{\circ} \\
\sin 45^{\circ} \cos 45^{\circ} & -\sin 45^{\circ} \cos 45^{\circ} \cos ^{2} 45^{\circ}-\sin ^{2} 45^{\circ}
\end{array}\right], \\
\mathbf{C}^{\prime}=\mathbf{T C T}^{T}=\left[\begin{array}{lll}
4.35 \times 10^{10} & 3.45 \times 10^{10} & 3.21 \times 10^{10} \\
3.45 \times 10^{10} & 4.35 \times 10^{10} & 3.21 \times 10^{10} \\
3.21 \times 10^{10} & 3.21 \times 10^{10} & 3.49 \times 10^{10}
\end{array}\right] .
\end{gathered}
$$

According to (15), we can get the material eigenvalues from $\operatorname{det} \mathbf{D}=0$ :

$$
\begin{gathered}
p_{1}=-0.934-0.358 i \\
p_{2}=0.348-0.937 i \quad p_{3}=i .
\end{gathered}
$$

According to (16) and (17), we can get material eigen matrixes $\mathbf{A}$ and $\mathbf{B}$

$$
\begin{gathered}
\mathbf{A}=\left[\begin{array}{ccc}
1.90 \times 10^{-6}+2.25 \times 10^{-6} i & -4.61 \times 10^{-6}-4.22 \times 10^{-6} i & 0 \\
2.65 \times 10^{-6}+1.09 \times 10^{-6} i & 4.22 \times 10^{-6}+4.61 \times 10^{-6} i & 0 \\
0 & 0 & 2.68 \times 10^{-6}-2.68 \times 10^{-6} i
\end{array}\right] \\
\mathbf{B}=\left[\begin{array}{ccc}
6.71 \times 10^{4}-4.61 \times 10^{4} i & -8.90 \times 10^{3}+4.96 \times 10^{4} i & 0 \\
4.61 \times 10^{4}-6.71 \times 10^{4} i & 4.96 \times 10^{4}-8.90 \times 10^{3} i & 0 \\
0 & 0 & 9.34 \times 10^{4}+9.34 \times 10^{4} i
\end{array}\right]
\end{gathered}
$$




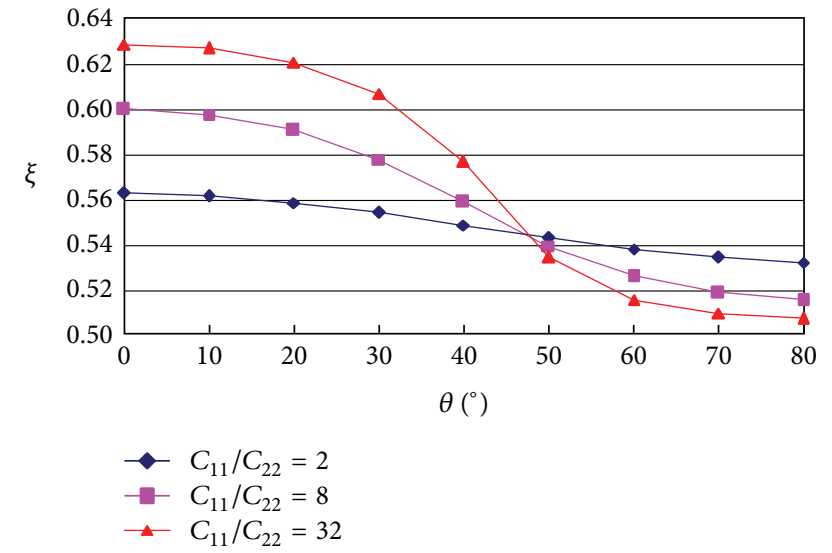

Figure 6: The first eigenvalue while $2 \alpha=270^{\circ}$.

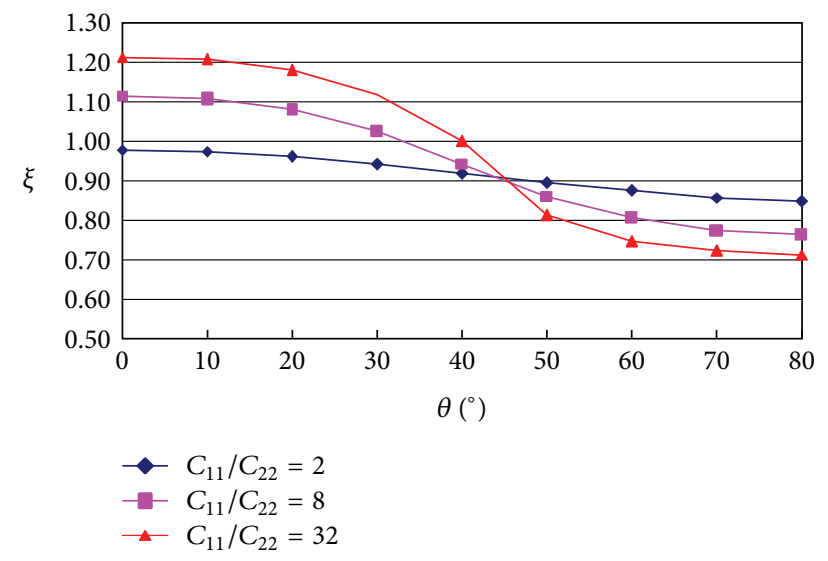

Figure 7: The second eigenvalue while $2 \alpha=270^{\circ}$.

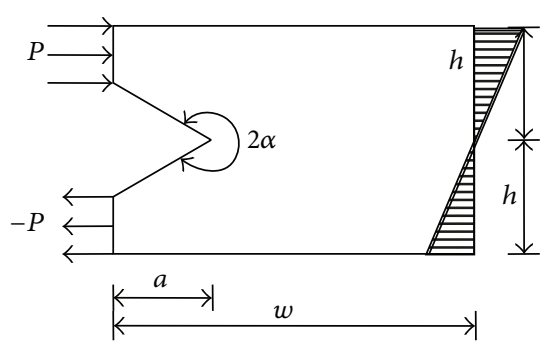

FIGURE 8: V-notch specimen under the antisymmetric load.

Substituting matrix B into (28) and (29), with the application of the modified Müller method SRAM, the characteristic eigenvalues of different V-notch angles $2 \alpha$ are showed in Table 2. $\xi_{1}$ and $\xi_{2}$ are the first and second eigenvalues, respectively.

6.2. Example 2. This example analyses the characteristic solutions of a V-notch and studies the influence of the material parameters, the angle of $2 \alpha$ in the V-notch, and the layer angle of $\theta$.
Tables 3, 4, and 5 study the first and the second characteristic eigenvalues $\left(\xi_{1}\right.$ and $\xi_{2}$ ) of three kinds of material $\left(C_{11} / C_{22}=2,8,32\right)$, eight types of V-notch $\left(2 \alpha=190^{\circ}, 200^{\circ}\right.$, $210^{\circ}, 240^{\circ}, 270^{\circ}, 300^{\circ}, 330^{\circ}, 360^{\circ}$ ), and eight types of layer angle $\left(\theta=10^{\circ}, 20^{\circ}, 30^{\circ}, 40^{\circ}, 50^{\circ}, 60^{\circ}, 70^{\circ}, 80^{\circ}\right)$.

Figures 4, 5, 6, and 7 show the first and the second items of the eigenvalues with different $2 \alpha$ and $\theta$.

6.3. Example 3. This example employs the proposed element ATF-VN to calculate the tip area of the V-notch, which is subjected to the antisymmetric load as shown in the Figure 8. $a=1, w=3, h=1$, elastic constant is $E=0.21 \times 10^{7}$, Poisson's ratio is $\mu=0.3$, the load sum is $P=1$, and the values of angle $2 \alpha$ are $330^{\circ}$ and $350^{\circ}$. Table 6 gives the eigenvalues of the Vnotch problem. Table 7 gives the values of KII calculated by ATF-VN involving different number $(N)$ of the items of the analytical trial functions.

\section{Conclusion}

In this paper, the eigenvalues of anisotropic material in plane V-notch problem are analyzed. The material characteristic matrix of anisotropic and boundary characteristic equations of plane problems with notch is derived. The eigenvalues of the V-notch anisotropic plane problem are calculated by the SRAM method. Numerical examples show that the presented SRAM method has advantages of fast convergence and high accuracy and is easy to implement. The proposed element ATF-VN based on the analytical trial functions provides good performance in the calculation of the stress field near the tip of the V-notch.

\section{Conflict of Interests}

The authors do not have any conflict of interests with the content of the paper.

\section{Acknowledgments}

This project was supported by the National Natural Science Foundation of China (no. 11272340), the National Basic Research Programs of China (no. 2010CB731503).

\section{References}

[1] M. Z. Wang, Advanced Elasticity, Peking University Press, Beijing, China, 2002, (Chinese).

[2] X. Fu, S. Cen, and Y. Long, "The Analytical Trial Function Method (ATFM) for finite element analysis of plane crack/notch problems," Key Engineering Materials, vol. 385-387, pp. 617-620, 2008.

[3] X. Fu and Y. Long, "Analysis of plane notch problems with analytical trial functions' method," Engineering Mechanics, vol. 20, no. 4, pp. 33-38, 2003 (Chinese).

[4] Z. Niu, C. Cheng, J. Ye, and N. Recho, "A new boundary element approach of modeling singular stress fields of plane V-notch problems," International Journal of Solids and Structures, vol. 46, no. 16, pp. 2999-3008, 2009. 
[5] Z. Niu, D. Ge, C. Cheng, J. Ye, and N. Recho, "Evaluation of the stress singularities of plane V-notches in bonded dissimilar materials," Applied Mathematical Modelling, vol. 33, no. 3, pp. 1776-1792, 2009.

[6] C. Cheng, Z. Niu, H. Zhou, and N. Recho, "Evaluation of multiple stress singularity orders of a V-notch by the boundary element method," Engineering Analysis with Boundary Elements, vol. 33, no. 10, pp. 1145-1151, 2009.

[7] X. C. Ping, M. C. Chen, and J. L. Xie, "Finite element analyses of singular stresses in tips of V-notched anisotropic plates," in Proceedings of the International Conference on Mechanical Engineering and Mechanics, vol. 1-2, pp. 1105-1111, Wuxi, China, 2007.

[8] A. N. Stroh, "Dislocations and cracks in anisotropic elasticity," vol. 3, pp. 625-646, 1958.

[9] A. N. Stroh, "Steady state problems in anisotropic elasticity," vol. 41, pp. 77-103, 1958.

[10] K. Wu and F. Chang, "Near-tip fields in a notched body with dislocations and body forces," Journal of Applied Mechanics, vol. 60, no. 4, pp. 936-941, 1998.

[11] C. Dongye and T. C. T. Ting, "Explicit expressions of BarnettLothe tensors and their associated tensors for orthotropic materials," Quarterly of Applied Mathematics, vol. 47, no. 4, pp. 723-734, 1989.

[12] B. Gross and A. Mendelson, "Plane elastostatic analysis of Vnotched plates," International Journal of Fracture Mechanics, vol. 8, no. 3, pp. 267-276, 1972.

[13] A. Carpinteri, M. Paggi, and N. Pugno, "Numerical evaluation of generalized stress-intensity factors in multi-layered composites," International Journal of Solids and Structures, vol. 43, no. 3-4, pp. 627-641, 2006.

[14] D. H. Chen, "Stress intensity factors for V-notched strip under tension or in-plane bending," International Journal of Fracture, vol. 70, no. 1, pp. 81-97, 1995.

[15] M.-C. Chen and K. Y. Sze, "A novel hybrid finite element analysis of bimaterial wedge problems," Engineering Fracture Mechanics, vol. 68, no. 13, pp. 1463-1476, 2001.

[16] M. Chen and X. Ping, "Finite element analysis of piezoelectric corner configurations and cracks accounting for different electrical permeabilities," Engineering Fracture Mechanics, vol. 74, no. 9, pp. 1511-1542, 2007. 


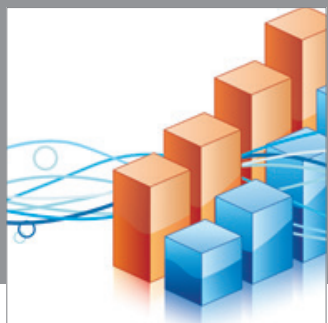

Advances in

Operations Research

mansans

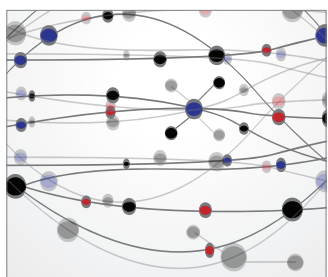

The Scientific World Journal
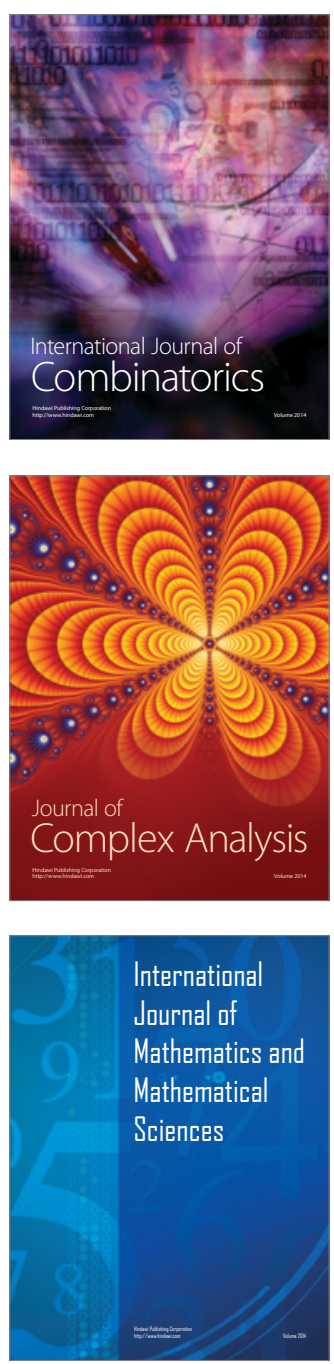
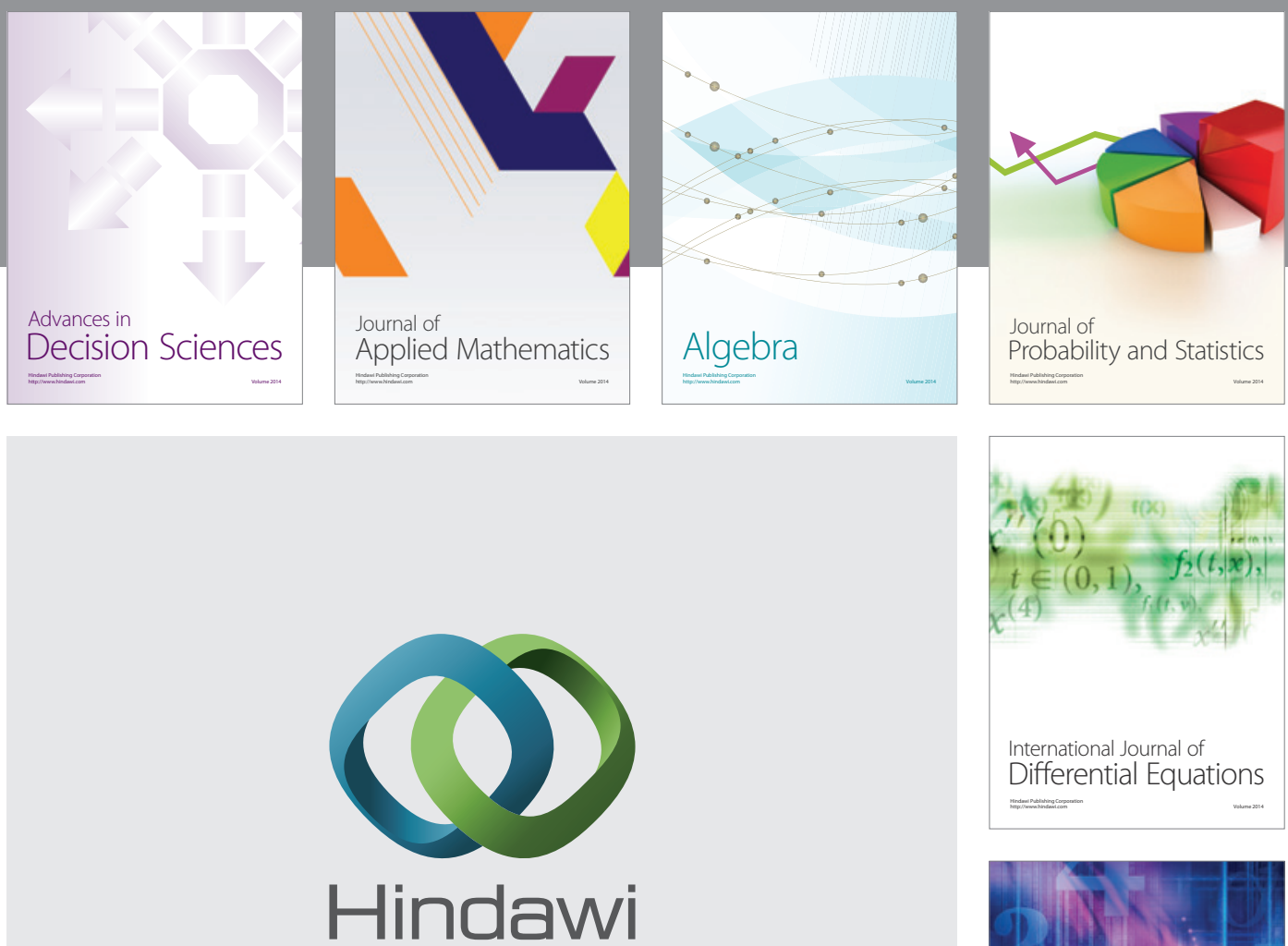

Submit your manuscripts at http://www.hindawi.com
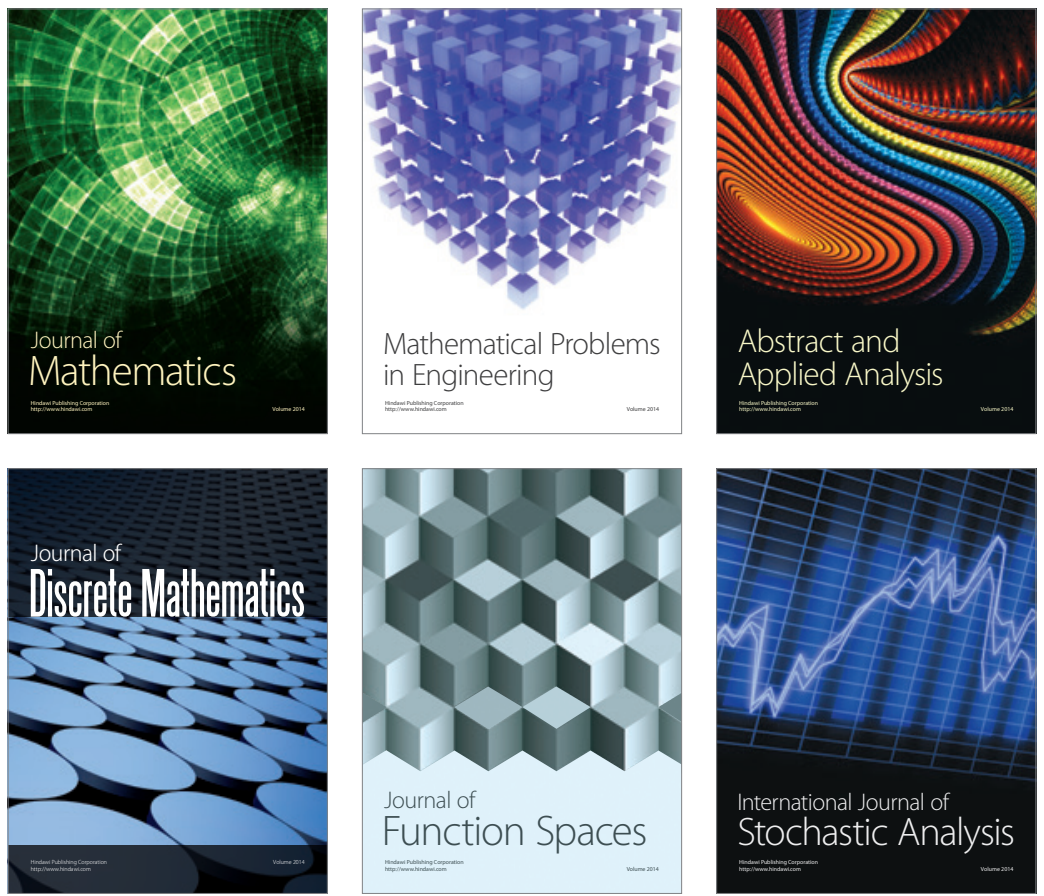

Journal of

Function Spaces

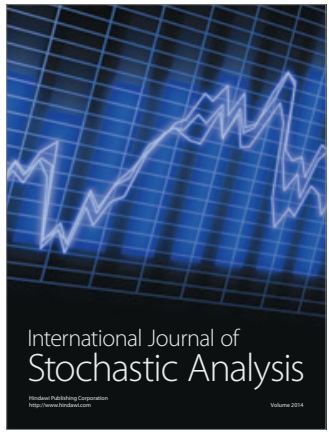

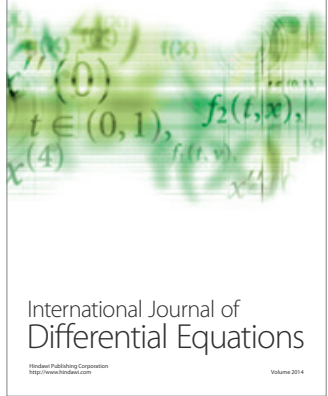
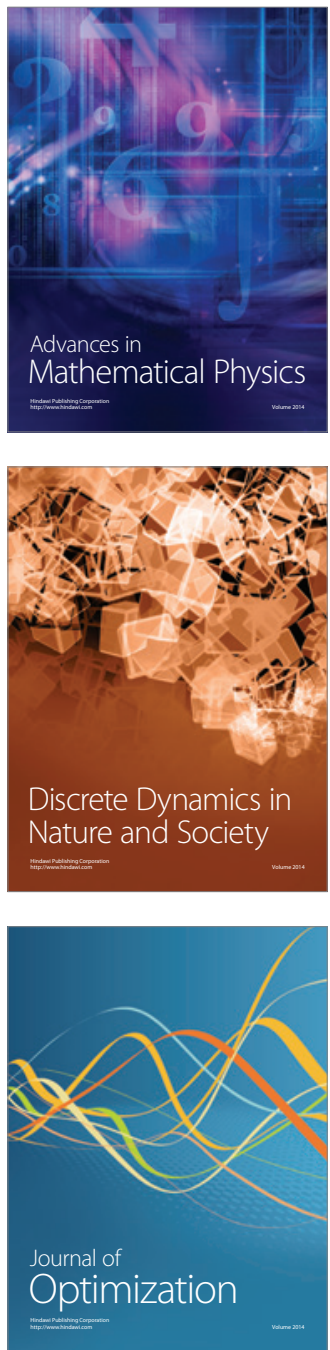